\title{
Acid-catalyzed hydrolysis of the vinyl ether group of 4-methoxy-1,2- dihydronaphthalene.Effect of conformation on reactivity
}

\author{
A. Awwal, ${ }^{1,2,3}$ W. E. Jones, ${ }^{2}$ A. J. Kresge, ${ }^{*}, 3$ and Q. Meng ${ }^{3}$ \\ Department of Chemistry, University of Toronto, Toronto, Ontario M5S 3H6, Canada and \\ Department of Chemistry and Biochemistry, \\ University of Windsor, Windsor, Ontario N9B 3P4, Canada \\ E-mail:akresge@chem.utoronto.ca
}

This paper is dedicated to Professor Dionis Sunko on the occasion of his $80^{\text {th }}$ birthday and in recognition of his contributions to physical organic chemistry

(received 09 Nov 01; accepted 04 Nov 01; published on the web 12 Nov 01)

\begin{abstract}
Rates of acid-catalyzed hydrolysis of the vinyl ether group of 4-methoxy-1,2-dihydronaphthalene were measured in dilute aqueous perchloric acid solutions and also in a series of carboxylic acid buffers. These data give the hydronium-ion isotope effect $k_{\mathrm{H}} / k_{\mathrm{D}}=3.39$ and provide a Brønsted relation with the exponent $\alpha=0.70$. The hydronium ion catalytic coefficient for this reaction is less, by a factor of 2.4, than that for the corresponding reaction of 3-methoxyindene. This rate difference is consistent with the coplanar arrangement of the vinyl and benzene groups in indene but non-coplanar disposition of these groups in dihydronaphthalene, and the consequent reduced ability of the benzene ring of dihydronaphthalene to stabilize the adjacent positive charge being put there in the rate-determining step of the vinyl ether hydrolysis rreaction.
\end{abstract}

Keywords: Hydrolysis, vinyl ethers, kinetics, dihydronaphthalene, conformation, Brønsted plot

\section{Introduction}

Indene, $\mathbf{1}$, is believed to be a planar molecule, ${ }^{4}$ in contrast to its next higher homolog, 1,2dihydronaphthalene, 2, whose nonaromatic ring is puckered and whose vinyl group is out of coplanarity with its benzene ring by some $15 .{ }^{5}$ 


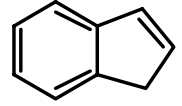

1

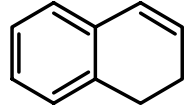

2

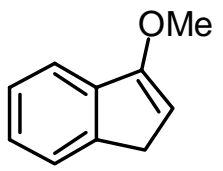

3<smiles>COC1=CCCc2ccccc21</smiles>

4

Rates of addition of electrophiles to the vinyl group of 1,2-dihydronaphthalene should consequently be lower than those of electrophilic addition to the vinyl group of indene. This follows from the fact that such addition generates positive charge on the vinyl carbon atom adjacent to the benzene rings of these molecules, where it can be stabilized by delocalization into these rings, and such stabilization is at its maximum when the vacant p-orbital of the carbon atom taking on this positive charge is parallel to the p-orbitals making up the benzene ring $\pi$ systems. In planar indene, this optimum configuration is already present in the initial state of the reaction, but in nonplanar 1,2-dihydronaphthalene it can be achieved only at the cost of opposing the forces that make this molecule nonplanar.

Acid-catalyzed vinyl ether hydrolysis is a reaction that occurs by rate-determining electrophilic addition of a proton to the $\beta$-carbon atom of the vinyl ether group, ${ }^{6}$ eq. 1 ,

$$
\mathrm{CH}_{2}=\mathrm{CHOR}+\mathrm{H}^{+} \rightarrow \mathrm{CH}_{3} \mathrm{CHOR}^{+}
$$

and the rate of this reaction for 3-methoxyindene has already been measured. ${ }^{7}$ We have consequently now carried out additional measurements of the hydrolysis of 4-methoxy-1,2dihydronaphthalene, $\mathbf{4}$, and have found that the rate difference predicted by the conformational argument made above is in fact realized.

\section{Experimental Section}

Materials. 4-Methoxy-1,2-dihydronaphthalene was prepared by treating $\alpha$-tetralone with trimethyl orthoformate in the presence of a catalytic amount of $p$-toluenesulfonic acid; ${ }^{8}$ its ${ }^{1} \mathrm{H}$ NMR spectrum agreed with a published report. ${ }^{9}$ All other materials were best available commercial grades.

Kinetics. Rates of hydrolysis of 4-methoxy-1,2-dihydronaphthalene were measured spectrophotometrically by monitoring either the decay of absorbance of this substance at $\lambda=222$ $\mathrm{nm}$ or the rise of absorbance of its hydrolysis product, $\alpha$-tetralone at $\lambda=254 \mathrm{~nm}$. The measurements were made using both conventional (Cary 2200 and Hewlett Packard 8453) and stopped-flow (Hi-Tech Scientific SF-S1) spectrometers, and the temperature of all reacting solutions was controlled at $25.0 \pm 0.05{ }^{\circ} \mathrm{C}$. Substrate concentrations in the reacting solutions were of the order of $10^{-5} \mathrm{M}$. The data so obtained conformed to the first-order rate law well, and observed first-order rate constants were obtained by least squares fitting of an exponential function. 


\section{Results}

Rates of hydrolysis of 4-methoxy-1,2-dihydronaphthalene were measured in aqueous $\left(\mathrm{H}_{2} \mathrm{O}\right.$ and $\mathrm{D}_{2} \mathrm{O}$ ) solutions of perchloric and hydrochloric acids over the concentration range $\left[\mathrm{L}^{+}\right]=0.001-$ $0.1 \mathrm{M}$ at a constant ionic strength of $0.10 \mathrm{M}$ maintained through the addition of sodium perchlorate as required. The data so obtained are displayed in Figure 1. It may be seen that observed first-order rate constants are accurately proportional to acid concentration, and the data were therefore analyzed by linear least squares fitting. This produced the hydronium-ion catalytic coefficient $k_{\mathrm{H}}=(2.43 \pm 0.01) \times 10^{1} \mathrm{M}^{-1} \mathrm{~s}^{-1}$ and the isotope effect $k_{\mathrm{H}} / k_{\mathrm{D}}=3.39 \pm 0.05$.

Rate measurements were also made in aqueous $\left(\mathrm{H}_{2} \mathrm{O}\right)$ carboxylic acid buffers using series of solutions of varying total buffer concentration but constant buffer ratio and constant ionic strength $(0.10 \mathrm{M})$. This served to keep hydronium ion concentrations along a given solution series constant for the most part. In some of the more acidic buffer solutions, however, "buffer failure" occurred, causing $\left[\mathrm{H}_{3} \mathrm{O}^{+}\right]$to drop as the buffer concentration was lowered, and in these cases appropriate adjustments were made. ${ }^{10}$

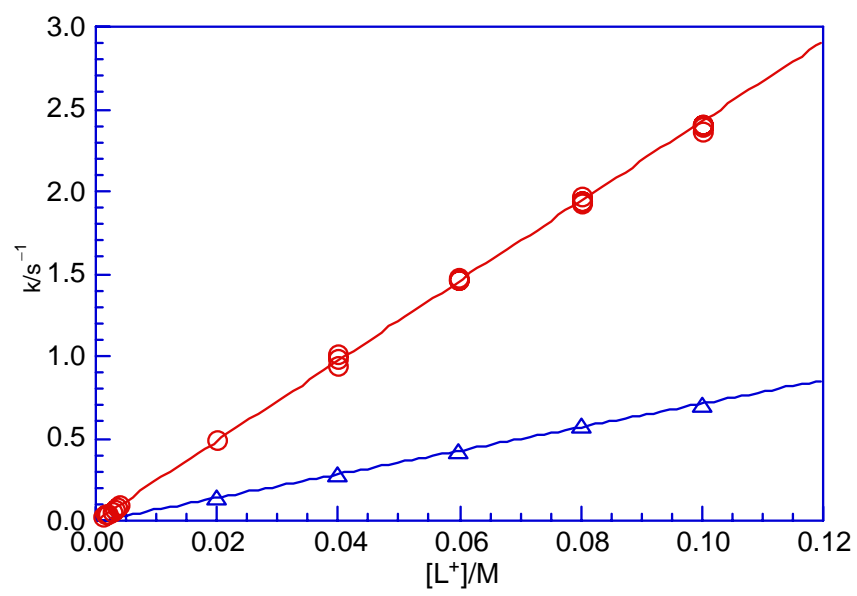

Figure 1. Rates of hydrolysis of 4-methoxy-1,2-dihydronaphthalene in aqueous acidic $\mathrm{H}_{2} \mathrm{O}(\mathrm{O})$ and $\mathrm{D}_{2} \mathrm{O}(\Delta)$ solutions at $25^{\circ} \mathrm{C}$.

Observed or adjusted first-order rate constants proved to be linear functions of buffer acid concentration, and general-acid catalytic coefficients, $k_{\mathrm{HA}}$, were determined by linear least squares analysis. The results obtained are listed in Table 1. 


\section{Discussion}

Table 1. General acid catalytic coefficients for the hydrolysis of 4-methoxy-1,2dihydronaphthalene in aqueous $\left(\mathrm{H}_{2} \mathrm{O}\right)$ solution at $25^{\circ} \mathrm{C}$

\begin{tabular}{lc}
\hline \multicolumn{1}{c}{$\mathrm{HA}$} & $k_{\mathrm{HA}} / 10^{-2} \mathrm{M}^{-1} \mathrm{~s}^{-1}$ \\
\hline $\mathrm{CNCH}_{2} \mathrm{CO}_{2} \mathrm{H}$ & 121 \\
$\mathrm{ClCH}_{2} \mathrm{CO}_{2} \mathrm{H}$ & 125 \\
$\mathrm{CH}_{3} \mathrm{OCH}_{2} \mathrm{CO}_{2} \mathrm{H}$ & 46.5 \\
$\mathrm{HCO}_{2} \mathrm{H}$ & 17.7 \\
$\mathrm{HOCH}_{2} \mathrm{CO}_{2} \mathrm{H}$ & 20.2 \\
$\mathrm{CH}_{3} \mathrm{CO}_{2} \mathrm{H}$ & 3.83 \\
$\mathrm{CH}_{3} \mathrm{CH}_{2} \mathrm{CO}_{2} \mathrm{H}$ & 3.72 \\
\hline
\end{tabular}

Reactions such as vinyl ether hydrolysis that occur by rate-determining proton transfer from a catalyzing acid to the substrate are expected to show hydronium-ion isotope effects in the normal direction $\left(k_{\mathrm{H}} / k_{\mathrm{D}}>1\right)$. The isotope effect determined here for the hydrolysis of 4-methoxy1,2-dihydronaphthalene, $k_{\mathrm{H}} / k_{\mathrm{D}}=3.39$, meets this expectation. It, in fact, agrees very well with such isotope effect determined for the hydrolysis of a group of some 30 other vinyl ethers. ${ }^{11}$ These other isotope effects were found to vary in a sensible fashion with vinyl ether reactivity and could be correlated using an expression based on Marcus rate theory. The correlation predicts $k_{\mathrm{H}} / k_{\mathrm{D}}=3.38$ for the present vinyl ether, in remarkably good agreement with the experimentally determined value.

Rate determining proton transfers from catalyst to substrate are also expected to conform to the Brønsted relation, ${ }^{12}$ which correlates catalytic coefficients with catalyst acid strength. Figure 2 shows that the present reaction fulfills this expectation as well, with general acid catalytic coefficients increasing regularily with acid strength. 


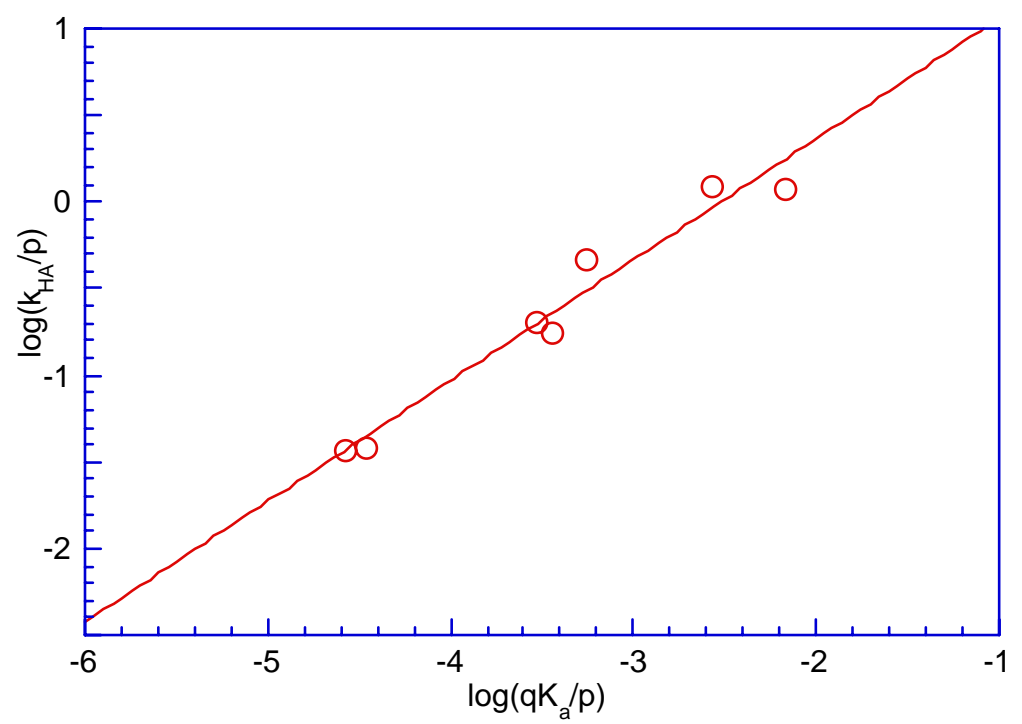

Figure 2. Brønsted plot for the hydrolysis of 4-methoxy-1,2-dihydronaphthalene in aqueous solution at $25^{\circ} \mathrm{C}$.

The Brønsted exponent given by this correlation is $\alpha=0.70 \pm 0.06$, which is a reasonable value for a substrate of the present reactivity. ${ }^{13}$ Fulfillment of these expectations, validates identification of the presently studied process as a vinyl ether hydrolysis reaction. Comparison of the hydronium-ion rate constant determined here with that reported for 3-methoxyindene, $k_{\mathrm{H}}=$ $55 \mathrm{M}^{-1} \mathrm{~s}^{-1}$, then shows the present reaction to be slower by a factor of 2.4 . This is consistent with the argument made above regarding the nonplanarity of dihydronaphthalene, in contrast to the planarity of indene, and the consequent reduced ability of the benzene ring of dihydronaphthalene to stabilize the positive charge generated by electrophilic addition to its vinyl group double bond. The factor of 2.4 found here is not large, but that is because most of the positive charge stabilization in the hydrolysis of these vinyl ethers is provided by the methoxy group, leaving little to be effected by the benzene rings. For example, comparison of the hydronium-ion catalytic coefficient for 3-methoxyindene with that for methyl vinyl ether, $k_{\mathrm{H}}=$ $0.76 \mathrm{M}^{-1} \mathrm{~s}^{-1}$, gives a phenyl group stabilization of only 76 fold.

\section{Acknowledgements}

We are grateful to the Natural Sciences and Engineering Research Council of Canada for financial support of this work. 


\section{References and Notes}

1. On leave from Jahangirnagar University.

2. University of Windsor.

3. University of Toronto.

4. (a) Diehl, P.; Vogt, J. J. Org. Magnetic Resonance 1975, 7, 81. (b) Southern, J. F.; Schäfer, L.; Brendenhaugen, K.; Seip, H. M. J. Chem. Phys. 1971, 55, 2418 and references cited therein.

5. Sygula, A.; Rabideau, P. W. J. Mol. Structure (Theochem) 1992, 262, 117.

6. Kresge, A. J. Acc. Chem. Res. 1987, 20, 364.

7. Chiang, Y.; Chwang, W. K.; Kresge, A. J.; Robinson, L. H.; Sagatys, D. S.; Young, C. I. Can J. Chem. 1978, 56, 456.

8. Rozen, S.; Mishani, E.; Kol, M. J. Am. Chem. Soc. 1992, 114, 7643.

9. Ortholand, J.-Y.; Vicart, N.; Greiner, A. J. Org. Chem. 1995, 60, 1880.

10. Keeffe, J. R.; Kresge, A. J. In Investigation of Rates and Mechanisms of Reactions Part 1; Bernasconi, C. F. Ed.; Wiley-Interscience, 1986; pp 776-778.

11. Kresge, A. J.; Sagatys, D. S.; Chen, H. L. J. Am. Chem. Soc. 1977, 99, 7228.

12. Brønsted, J. N.; Pedersen, K. Z. Phys. Chem. 1924, 108, 185.

13. (a) Kresge, A. J. Chem. Soc. Rev. 1973, 2, 475. (b) Kresge, A. J.; Chen, H. L; Chiang, Y.; Murrill, E.; Payne, M. A.; Sagatys, D. S. J. Am. Chem. Soc. 1971, 93, 413. 